Check for updates

Cite this: RSC Adv., 2019, 9, 25730

Received 11th July 2019

Accepted 11th August 2019

DOI: $10.1039 / \mathrm{c} 9 \mathrm{ra05220h}$

rsc.li/rsc-advances

\section{Preparation of highly interconnected porous polymer microbeads via suspension polymerization of high internal phase emulsions for fast removal of oil spillage from aqueous environments $\uparrow$}

\author{
Xiaoling Cui, ${ }^{a}$ Hong Shao, ${ }^{a}$ Yuanrui Song, ${ }^{a}$ Song Yang, ${ }^{b}$ Fengwei Wang ${ }^{c}$ \\ and Huarong Liu (iD *a
}

In this paper, we report on the fabrication of highly interconnected porous poly(butyl acrylate-co-stearyl methacrylate-co-styrene-co-divinylbenzene) (P(BA-SMA-St-DVB)) monoliths and microbeads via the polymerization of high internal phase emulsions (HIPEs) and double emulsions by using a $75 \%$ to $85 \%$ internal water phase. The morphology and specific surface area were characterized with scanning electron microscopy (SEM) and nitrogen adsorption/desorption measurements, respectively. The oil absorbency, including oil absorption, oil absorption rate and oil retention ratio of as-prepared resins (both monoliths and microbeads), was studied by using kerosene and carbon tetrachloride as analogues of oil pollutants. Thermoanalysis was also carried out to analyze the oil absorption mechanism. The results showed that the resin had a good oil absorption efficiency of aliphatic hydrocarbon and chlorohydrocarbon. The best massbased absorption capacity of the microbeads towards kerosene and carbon tetrachloride is 8.52 and 20.82 times its own weight, respectively, which is higher than those of the corresponding polyHIPE monoliths. Meanwhile, these microbeads of average 320 microns in diameter have good thermal stability and can be easily collected and recycled by a simple filtration washing method.

\section{Introduction}

Nowadays, crude oil spill accidents have been recognized as one of most severe environmental problems due to their toxic effects on marine aquatic flora and fauna, as well as other adverse ecological impacts., ${ }^{\mathbf{1 , 2}}$ Moreover, the increasing industrial oilpolluted wastewater has also become a great challenge around the world. ${ }^{3,4}$ So there is an increasing demand to develop oilwater separation technologies for the collection and elimination of oils or organic contaminants from polluted water with high efficiency. ${ }^{5-7}$ At present, several main techniques for oilwater separation have been developed, such as oil collection machines, ${ }^{8}$ in situ burning, ${ }^{\mathbf{9}, 10}$ bioremediation with microorganisms, ${ }^{\mathbf{1 1 , 1 2}}$ chemical treatment with dispersants and detergents $^{\mathbf{1 3}}$ and oil absorption materials. ${ }^{\mathbf{1 4}}$ Among these

${ }^{a}$ CAS Key Laboratory of Soft Matter Chemistry, Department of Polymer Science and Engineering, University of Science and Technology of China, Hefei, Anhui 230026, P. R. China.E-mail: hrliu@ustc.edu.cn

${ }^{b}$ Key Laboratory of Tobacco Chemistry, Zhengzhou Tobacco Research Institute of CNTC, Zhengzhou, Henan 450001, P. R. China

${ }^{c}$ Department of Electronic Packaging Technology, School of Materials Science and Technology, Jiangsu University of Science and Technology, Zhenjiang, Jiangsu, 212003, P. R. China

$\dagger$ Electronic supplementary information (ESI) available. See DOI: 10.1039/c9ra05220h technologies, adsorption by oil absorbents is considered to be one of the most efficient techniques, and has distinct advantages of simple operation, low cost, and complete cleanup without secondary pollution. ${ }^{15-18}$

Interconnected porous resins with hydrophobicity and lipophilicity are preferential candidates for oil absorption because of their outstanding oil-water selectivity, high absorption capacities, fast absorption kinetics, excellent reusability, and oil recovery. ${ }^{19}$ As is known to all, the templating of high internal phase emulsions (HIPE) is an effective method to prepare highly interconnected porous polymeric materials, often referred to as polyHIPEs, which possess ultra-high permeability. ${ }^{20}$ The emulsions consist of an external continuous monomer phase and an internal droplet phase with volume fraction of at least $0.74 .^{21,22}$ This HIPE templating method is flexible and easy to control pore structure and size as well as porosity by varying the compositions of the emulsions, compared with other methods for the fabrication of porous polymers. ${ }^{23-25}$ In previous reports, polyHIPEs are fabricated by various monomer precursors, such as styrene, ${ }^{26,27} 4$-vinylbenzyl chloride ${ }^{28}$ methyl methacrylate, ${ }^{27,29}$ 2-hydroxyethyl methacrylate, ${ }^{30}$ 2-ethylhexyl acrylate, ${ }^{31}$ stearyl methacrylate, ${ }^{32}$ hexafluorobutyl acrylate, ${ }^{33}$ butyl acrylate, ${ }^{34}$ and so on, giving mechanical properties that range from elastomeric to extremely tough and rigid. ${ }^{35}$

Because of their high porosity, large surface area, open and interconnected pores, polyHIPEs have great potential in the 
field of separation. At present, the reported polyHIPEs materials used for oil absorption are all monoliths. For example, Fang's group reported that ultra-low density porous polystyrene ${ }^{36}$ and porous polymeric composite monoliths, ${ }^{37}$ which were prepared via using W/O HIPEs as templates stabilized by a low-molecular mass gelator, can be used as excellent absorbents for many organic liquids. Zhang et al. described superhydrophobic P(StDVB) foam prepared by the HIPE technique introduced with $\mathrm{Fe}_{3} \mathrm{O}_{4}$ nanoparticles for oil removal applications. ${ }^{38,39}$ Later, they reported spongy polystyrene by dual-templating synthesis for oil capture with excellent adsorption properties. ${ }^{40}$ Guo's group described a novel strategy to prepare HIPE organogels by charge-driven assembly of a triblock ionomer and an inorganic nanoparticle ${ }^{41}$ or a polypropylenimine dendrimer, ${ }^{42}$ which can be used as oil absorbent. Zhou and coworkers prepared closedcell high-porosity supermacroporous polystyrene monoliths with high oil-water separation property via water-in-oil Pickering high internal phase emulsions by employing amphiphilic carbonaceous microspheres (CMs) as stabilizer without any inorganic salts. ${ }^{43}$ Guo et al. reported macroporous monoliths with $\mathrm{pH}$-induced switchable wettability for recyclable oil separation and recovery. ${ }^{\mathbf{4 4}}$

However, there were no reports on the application of polyHIPE microbeads for oil absorption as far as we know. Compared with polyHIPE monoliths, polyHIPE microbeads of submillimeter can absorb oil faster due to a larger contact area with the oil, and also regenerate them more easily for recycling. Generally, polyHIPE microbeads were fabricated by using oil-inwater-in-oil $(\mathrm{O} / \mathrm{W} / \mathrm{O})$ sedimentation polymerization ${ }^{\mathbf{4 5 4}}$ and water-in-oil-in-water $(\mathrm{W} / \mathrm{O} / \mathrm{W})$ suspension polymerization. ${ }^{\mathbf{4 7 - 4 9}}$ Recently, a microfluidic setup was used to fabricate polyHIPE microbeads of diameters around $400 \mu \mathrm{m} .^{\mathbf{5 0}}$

Herein, we fabricate highly interconnected porous polymeric monoliths and microbeads by using butyl acrylate, stearyl methacrylate and styrene as co-monomers and divinylbenzene as cross-linker via HIPE templating and double emulsion templating, respectively, to compare their adsorption efficiencies and capacities. Butyl acrylate is selected as a comonomer to both introduce carboxyl group and increase the flexibility of polyHIPEs; and stearyl methacrylate (SMA) is found to be useful in separation process and oil absorption due to the long C18 side chain that can move freely to a certain extent. ${ }^{32,51}$ The opencell polyHIPEs microbeads are first used as oil-absorption resins, and their oil-absorption mechanism is studied by thermoanalysis.

\section{Experimental section}

Materials

Stearyl methacrylate (SMA, 96\%, technical grade) and divinylbenzene (DVB, $80 \%$, technical grade, with the rest $20 \%$ of diethylbenzene) were purchased from Sigma-Aldrich. DVB was used as received and SMA was purified by recrystallization with ethanol for twice. Kerosene were obtained from Aladdin Reagent Co. Ltd., (China), while carbon tetrachloride $\left(\mathrm{CCl}_{4}\right)$ and other common chemical reagents were purchased from Sinopharm Chemical Reagents Co., Ltd. (China). Styrene (St, 99\%) and $n$-butyl acrylate (BA, 98\%) were purified to remove the inhibitor by passing through a basic alumina column before use. Azo-bis-isobutyronitrile (AIBN) and potassium persulphate (KPS) were used after recrystallization with ethanol. Sorbitan monooleate (Span 80) and poly( $N$-vinyl-pyrrolidone) (PVP, $\mathrm{K}_{30}$, $M_{\mathrm{w}} \sim 40000 \mathrm{~g} \mathrm{~mol}^{-1}$ ) were of reagent grade and used without further purification. 12-Acryloxy-9-octadecenoic acid (AOA) was commercial available from USTC Chuangxin Co., Ltd. (China). Double-distilled and deionized (DI) water was used in all experiments.

\section{Preparation of high internal phase emulsion and monolith resins}

A schematic illustration of the preparation of polyHIPE microbeads is shown in Scheme 1. In our experiments, a mixture of styrene $(0.30 \mathrm{~g})$, butyl acrylate $(0.60 \mathrm{~g})$, stearyl methacrylate $(0.60 \mathrm{~g})$, divinylbenzene $(0.50 \mathrm{~g})$, Span $80(0.30 \mathrm{~g})$ and AIBN (0.04 g) were used as oil phase (O). The oil phase was placed into a $50 \mathrm{ml}$ beaker, then a certain amount of aqueous solution $\left(\mathrm{W}_{1}\right)$ containing $2 \mathrm{wt} \% \mathrm{NaCl}$ and $1 \mathrm{wt} \%$ KPS was added dropwise into the oil phase under stirring at $300 \mathrm{rpm}$. After the complete addition of the aqueous solution, the mixture was stirred for further $30 \mathrm{~min}$ to allow better homogenization of the HIPE. All the experimental ingredients in this paper are listed in Table 1. The as-prepared HIPEs were transferred to test tubes and polymerized into a shaped monoliths at a $60{ }^{\circ} \mathrm{C}$ water bath for $20 \mathrm{~h}$. The polyHIPE monoliths of poly(SMA-BA-St-DVB) with a diameter of $15 \mathrm{~mm}$ and a length of $30 \mathrm{~mm}$ were purified by Soxhlet extraction with ethanol and dried in a vacuum oven at $50{ }^{\circ} \mathrm{C}$ to constant weight. These samples were labeled as $1-\mathrm{m}-$ $75 \%, 2-\mathrm{m}-80 \%$ and $3-\mathrm{m}-85 \%$, respectively, according to their weight fraction of water phase.

\section{Preparation of double emulsion and microbead resins}

In order to prepare $\left(\mathrm{W}_{1} / \mathrm{O} \mathrm{HIPE}\right) / \mathrm{W}_{2}$ double emulsions, an outer aqueous phase $\left(\mathrm{W}_{2}\right)$ was prepared in advance with $2 \mathrm{~g}$ of PVP dissolved in $120 \mathrm{~g}$ of water. The $\mathrm{W}_{2}$ aqueous phase was put into a $250 \mathrm{ml}$ three-necked flask equipped with a reflux condenser and nitrogen inlet system which was placed in an oil bath of $120{ }^{\circ} \mathrm{C}$. The above HIPEs were respectively dropped into the boiling $\mathrm{W}_{2}$ phase by a syringe pump at a rate of flow of 5 $\mathrm{ml} \min ^{-1}$ under vigorous stirring. The reaction was continued for $6 \mathrm{~h}$ to ensure complete conversion. The microbeads were collected by filtration and washed with hot ethanol three times,

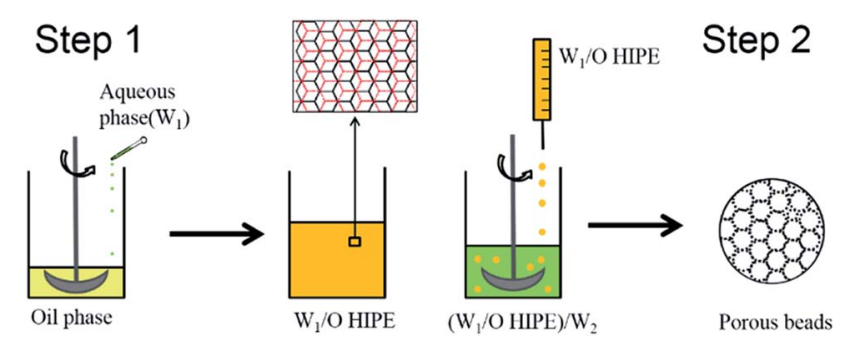

Scheme 1 Schematic illustration of preparation procedures of waterin-oil high internal phase emulsion (W $\left.\mathrm{W}_{1} / \mathrm{O} \mathrm{HIPE}\right)$ and $\left(\mathrm{W}_{1} / \mathrm{O} \mathrm{HIPE}\right) / \mathrm{W}_{2}$ double emulsion. 
Table 1 Ingredients of $W_{1} / O$ HIPEs and $\left(W_{1} / O\right.$ HIPE)/W $W_{2}$ double emulsions

\begin{tabular}{|c|c|c|c|c|c|c|c|c|c|c|c|}
\hline \multirow[b]{2}{*}{ Samples } & \multicolumn{3}{|c|}{$\mathrm{W}_{1}$ phase $(\mathrm{g})$} & \multicolumn{6}{|c|}{ O phase $(\mathrm{g})$} & \multicolumn{2}{|c|}{$\begin{array}{l}W_{2} \text { phase } \\
(\mathrm{g})\end{array}$} \\
\hline & $\mathrm{H}_{2} \mathrm{O}$ & KPS & $\mathrm{NaCl}$ & St & BA & SMA & DVB & AIBN & Span 80 & $\mathrm{H}_{2} \mathrm{O}$ & PVP \\
\hline $1-75 \%$ & 6.92 & 0.07 & 0.14 & 0.30 & 0.60 & 0.60 & 0.50 & 0.04 & 0.30 & 120 & 2 \\
\hline $2-80 \%$ & 9.10 & 0.09 & 0.18 & 0.30 & 0.60 & 0.60 & 0.50 & 0.04 & 0.30 & 120 & 2 \\
\hline 3-85\% & 12.65 & 0.13 & 0.25 & 0.30 & 0.60 & 0.60 & 0.50 & 0.04 & 0.30 & 120 & 2 \\
\hline
\end{tabular}

and then dried in a vacuum oven at $50{ }^{\circ} \mathrm{C}$ to constant weight. These samples were labeled as 1-b-75\%, 2-b-80\% and 3-b-85\%, respectively.

\section{Characterization}

Morphology and chemical structure. Fourier transform infrared (FTIR) spectra were taken on a Bruker VECTOR-22 FTIR spectrometer using the $\mathrm{KBr}$ pellet method. The morphology of highly interconnected porous microbeads and monoliths was observed on JEOL JSM-6700F field emission scanning electron microscopy (FESEM) at an operating voltage of $5 \mathrm{kV}$. Samples were prepared at room temperature by gluing the dry macroporous microbeads and monoliths onto conductive adhesive tapes, followed by $120 \mathrm{~s}$ of gold sputtering to ensure good electrical conductivity.

Specific surface area. Nitrogen adsorption/desorption measurements were performed at $77.3 \mathrm{~K}$ on a Micromeritics ASAP 2020 gas adsorption analyzer. Samples were degassed at $70{ }^{\circ} \mathrm{C}$ overnight under vacuum prior to data collection. The specific surface areas were collected over $0.05-0.30 P / P_{0}$ and calculated using the Brunauer-Emmett-Teller (BET) method. Pore volumes were determined from the amount of $\mathrm{N}_{2}$ adsorbed at $P / P_{0}=0.972$.

Oil-absorption capacity and oil-absorption rate. Oil absorption properties of macroporous monoliths and microbeads were determined using gravimetric analysis. PolyHIPE microbeads were enveloped in a tiny nylon bag and immersed into oil (kerosene or $\mathrm{CCl}_{4}$ ) for $20 \mathrm{~min}$ at room temperature. Then the bag was taken out and put onto a glass funnel to drain for $10 \mathrm{~min}$. The weight was recorded by an electrical balance. Such test was repeated until constant weight. Contrast experiments were taken out with the same procedure without the addition of polymeric microbead samples. The oil-absorption capacity was calculated by the following equation:

$$
\text { Oil-absorption capacity }\left(\mathrm{g} \mathrm{g}^{-1}\right)=\frac{W_{1}-W_{2}-W_{0}}{W_{0}}
$$

where $W_{0}$ is the original weight of polymeric microbeads, $W_{1}$ is the total weight of nylon bag and polymeric microbeads after immersed in oil and oil drainage, $W_{2}$ is the weight of nylon bag after oil drainage in contrast experiments.

For oil absorption capacity test of polyHIPE monoliths, the samples were immersed into oil for first $15 \mathrm{~min}$ and then were weighted every $10 \mathrm{~min}$. The samples were all wiped with filter paper to remove redundant oil sticking to the sample surface. The oil absorption capacity was determined by the following equation:

$$
\text { Oil-absorption capacity }\left(\mathrm{g} \mathrm{g}^{-1}\right)=\frac{W_{3}-W_{0}}{W_{0}}
$$

where $W_{0}$ is the original weight of polyHIPE monolith, $W_{3}$ is the weight of monolith absorbed with oil.

The oil-absorption rate was determined by oil-absorption capacity as a function of absorption time.

Oil retention ratio. The fully swollen resins by oil were put into an electric blast drying oven at $40{ }^{\circ} \mathrm{C}$ for $5 \mathrm{~min}, 15 \mathrm{~min}$ or $30 \mathrm{~min}$, and then these samples were weighted. The oil retention ratio was calculated according to the following equation:

$$
\text { Oil retention ratio }(\%)=\frac{W_{5}-W_{0}}{W_{4}-W_{0}} \times 100 \%
$$

where $W_{0}$ is the original weight of the dry microbeads or monoliths, $W_{4}$ and $W_{5}$ are the net weight of fully swollen resins before and after baking, respectively.

Cleanup of oil slick. The absorption ability toward spilled oil was tested under simulative conditions. DI water $(60 \mathrm{ml})$ was placed in a culture dish $(9 \mathrm{~cm}$ in diameter with the water-air surface of about $64 \mathrm{~cm}^{2}$ ), and then $0.36 \mathrm{~g}$ of kerosene was added on the surface of water as simulative oil slick. In order to characterize the cleanup efficiency, $0.06 \mathrm{~g}$ of oil-absorption resin, that would reach $80 \%$ absorption capacity in case of complete removal of oil spill, was added into the culture dish. The polymeric resin was kept floating calmly on the surface of the liquid for 10 minutes. The digital photos were taken as evidence.

Thermoanalysis. The microbeads fully swollen with kerosene were put into an electric blast drying oven at $40{ }^{\circ} \mathrm{C}$ for $2 \mathrm{~h}$ to reach constant weight. Then thermogravimetric analysis (TGA) was performed with a SDTQ600 TG-DTA Analyzer. Samples were heated in $\mathrm{N}_{2}$ atmosphere from 20 to $600{ }^{\circ} \mathrm{C}$ at a rate of $10{ }^{\circ} \mathrm{C} \min ^{-1}$.

\section{Results and discussion}

In this work, water-in-oil high internal phase emulsion $\left(\mathrm{W}_{1} / \mathrm{O}\right.$ HIPE) with an internal phase weight ratio of $75 \%, 80 \%$ and $85 \%$ were first prepared using styrene (St), butyl acrylate (BA) and stearyl methacrylate (SMA) as comonomers and divinylbenzene (DVB) as crosslinking agent and Span 80 as the emulsifier. The HIPEs were then put into external water phase $\left(\mathrm{W}_{2}\right)$ under stirring at $300 \mathrm{rpm}$ in the presence of poly( $N$-vinyl-pyrrolidone) (PVP) to form ( $\mathrm{W}_{1} / \mathrm{O}$ HIPE) $/ \mathrm{W}_{2}$ double emulsion. Macroporous $\mathrm{P}(\mathrm{BA}-\mathrm{SMA}-\mathrm{St}-\mathrm{DVB})$ monoliths and microbeads with highly interconnected porous structure were obtained by the polymerization of oil phase in HIPEs and $\mathrm{W} / \mathrm{O} / \mathrm{W}$ double emulsions 
(namely suspension polymerization of HIPEs via a double emulsion route), respectively. The FTIR spectrum of P(BA-SMASt-DVB) is illustrated in Fig. 1, which clearly demonstrates various chemical components. Specifically, the bands at 830 , 793, 762 and $699 \mathrm{~cm}^{-1}$ are attributed to the out-of-plane bending vibration of $\mathrm{C}-\mathrm{H}$ on the benzene ring, and those at 1602,1492 and $1452 \mathrm{~cm}^{-1}$ can be assigned to benzene ring vibrations. The bands at 3085,3057 and $3027 \mathrm{~cm}^{-1}$ are attributed to the stretching vibration of unsaturated $\mathrm{C}-\mathrm{H}$ on the benzene ring, while those at 2954, 2928 and $2854 \mathrm{~cm}^{-1}$ are assigned to the stretching vibration of saturated $\mathrm{C}-\mathrm{H}$ on the polymer backbone and pendant group of poly(meth)acrylates. Moreover, the peak at $1732 \mathrm{~cm}^{-1}$ corresponds to the stretching vibration of the carbonyl group, and that at $1161 \mathrm{~cm}^{-1}$ is owing to the stretching vibration of the $\mathrm{C}-\mathrm{O}-\mathrm{C}$ bond. Overall, the FTIR spectrum presents the chemical information of $\mathrm{P}(\mathrm{BA}-\mathrm{SMA}-\mathrm{St}-$ DVB).

The obtained P(BA-SMA-St-DVB) has a highly crosslinked structure, which retained high gel fraction (about 99\% wt) when extracted in good solvents, such as dichloroethane, as shown in Table S1 (ESI $\dagger$ ). In contrast, if without DVB, the obtained product was fully soluble in dichloroethane (Fig. S1 in the ESI $\dagger$ ). These results prove the formation of network structure for P(BA-SMA-St-DVB).

\section{Morphology of highly interconnected macroporous monoliths and microbeads}

The surface and inner structure of as-prepared microbeads as well as the inner structure of monoliths with different weight fractions of the internal water phase were characterized by SEM shown in Fig. 2. It can be found that the microbeads are ideal spherical with a diameter ranging from $30 \mu \mathrm{m}$ to $500 \mu \mathrm{m}$ (Fig. 2(A-C)). Moreover, the inner structure of microbeads exhibits a typical polyHIPE one as the same as shown in Fig. 2(G-I) of polyHIPE monolith samples, in which interconnected pores and pore throats are completely preserved (see from the occasional cracked surface of the microbeads in

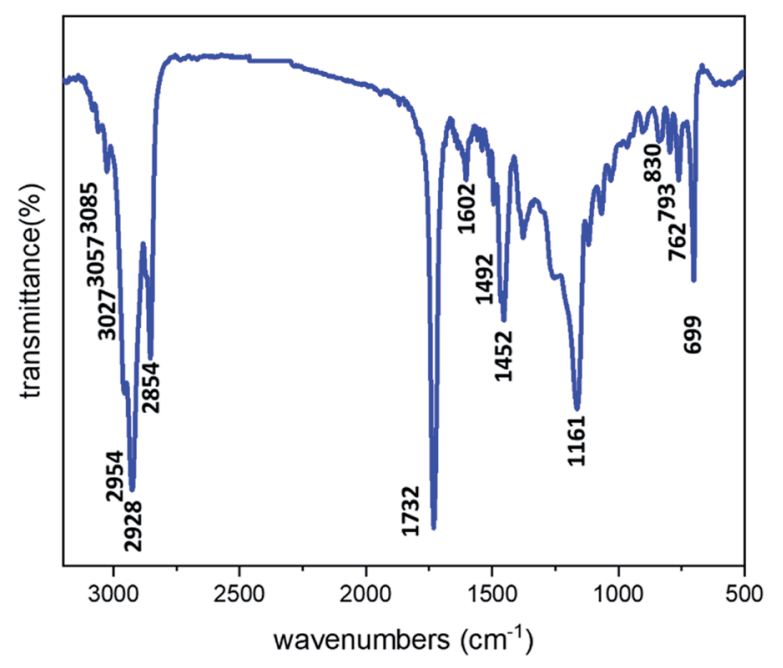

Fig. 1 The FTIR spectrum of $2-m-80 \%$ sample.
Fig. 2(D-F)). However, it is somewhat disappointed that the microbeads are not monodisperse, because the dispersion of HIPEs in water is not easy due to the high viscosity of HIPE which increases greatly with the increased weight fraction of the internal water phase. As a consequence, the particle size of the as-prepared polyHIPE microbeads increases with the increase of the weight fraction of the internal water phase under the same stirring speed of 300 rpm (see Fig. 2).

\section{Specific surface area}

One of the outstanding properties of highly interconnected macroporous microbeads and monoliths is the considerable specific surface area. As previous reported, polyHIPE monoliths generally own a specific surface area of $3-20 \mathrm{~m}^{2} \mathrm{~g}^{-1} .{ }^{21}$ The specific surface area and pore volume data of as-prepared polyHIPE microbeads and monoliths are summarized in Table 2. It can be found that the specific surface areas of microbeads are approximately the same as those of the corresponding monolith materials, and both of them slightly increase with the increase of weight fraction of the internal water phase. These specific surface areas are all around $3-6 \mathrm{~m}^{2} \mathrm{~g}^{-1}$, falling into the range of a typical value of polyHIPE foams. The nitrogen absorptiondesorption isotherm with a typical sample $2-b-80 \%$ is shown in Fig. 3, which exhibits a type II isotherm of macroporous materials in the IUPAC classification and a type H3 hysteresis loop. This result is consistent with the SEM image without the generation of mesopores in the sketch of polyHIPE.

\section{Oil-adsorption capacity}

The oil-absorption capacity is determined by diffusion coefficient of oils, van der Waals force between oil and polymer segments, as well as the structure of resins. ${ }^{52}$ When porous polymer resins contact with the water/oil mixture, oil penetrates into the resins owing to capillary force, and then replaced air and filled the vacancy because of its low surface tension. In this work, kerosene and carbon tetrachloride are used as different oil specimens to test the oil-absorption capacity of highly interconnected macroporous microbeads and monoliths by immersing the resin samples into oil at a certain time intervals using gravimetric analysis, and the results are collected in Fig. 4 and 5 as well as in Table 3. It can be seen from Fig. 4 that the macroporous microbeads could get saturated absorption of kerosene within about $120 \mathrm{~min}$ and the oil-absorption ratio reaches as much as $80 \%$ in the first 15 minutes. As a contrast, the saturated absorption of kerosene by monolith resin samples is achieved about 6-8 hours, with an absorption ratio of $75-80 \%$ in the first 15 minutes. Obviously, the microbeads can reach the saturation adsorption equilibrium more easily than the monoliths, which may be due to the larger contact area of microbeads with the oils. Of course, both microbeads and monoliths have a fast initial absorption velocity, which should be attributed to the highly interconnected macropores of typical HIPE structures that can enhance the mass transportation.

Due to a good swelling performance of aromatic polymer chains in carbon tetrachloride, these macroporous microbeads and monoliths fabricated by HIPE template method have both 

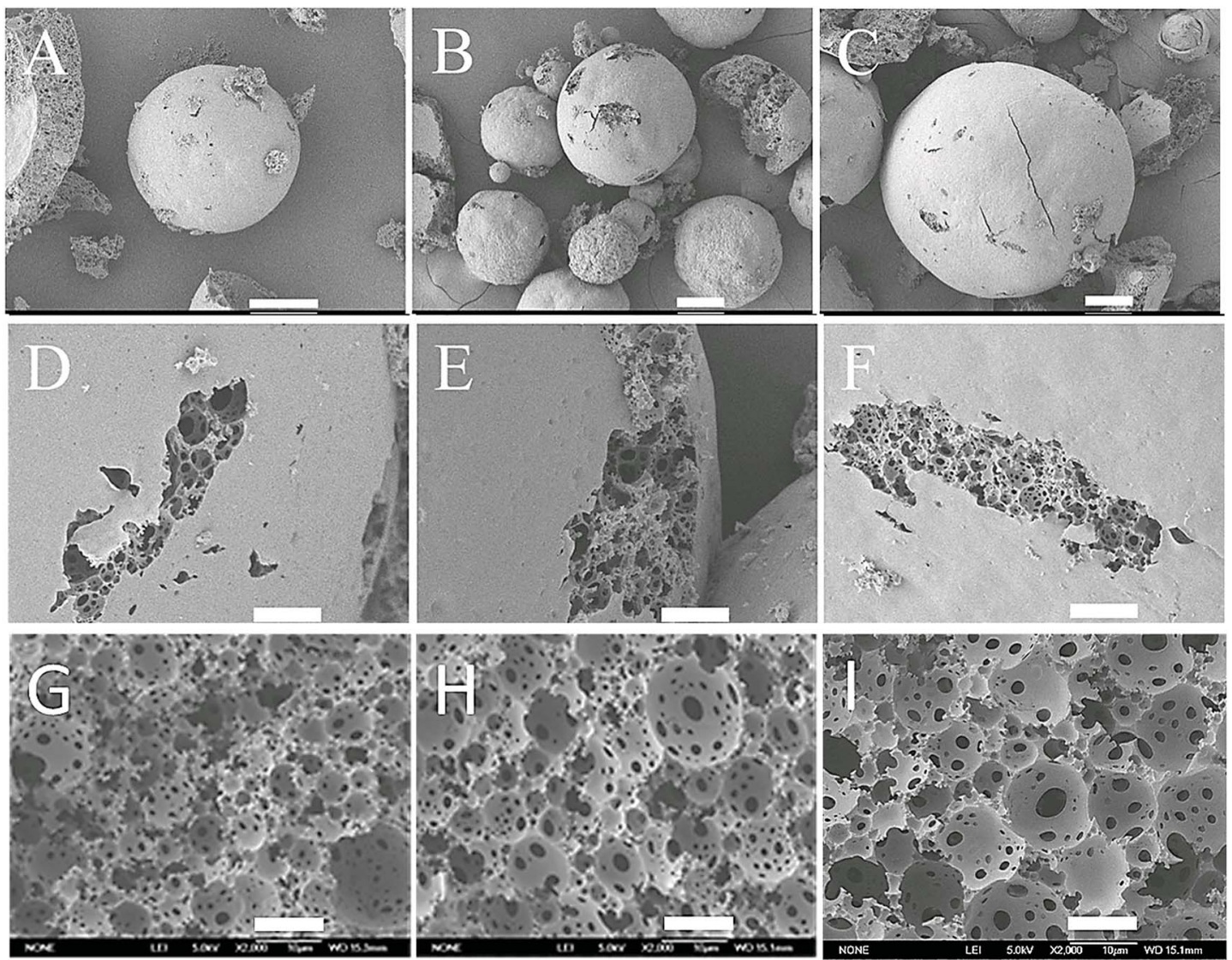

Fig. 2 SEM images of the appearance $(A-C)$ and the inner structure of the microbeads $(D-F)$ and monoliths $(G-I)$ obtained with different weight fraction of the internal water phase: (A, D and G) 75\%, (B, E and H) $80 \%,(C, F$ and I) $85 \%$. Scale bar: (A-C) $100 \mu \mathrm{m},(D-I) 10 \mu \mathrm{m}$.

a fast absorption velocity and a high oil-absorption ratio to carbon tetrachloride. As shown in Fig. 5, the absorption equilibrium can be reached in less than 30 minutes for both monolith and microbead samples. The oil absorption ratios of different microbead and monolith polymer resins are listed in Table 3. It can be found that macroporous microbeads have larger oil-absorption ratios than the corresponding monoliths to both kerosene and carbon tetrachloride at the same weight fraction of internal water phase $\left(\mathrm{W}_{1}\right)$, which may also be due to the larger contact area and the larger effective pore volume of microbeads to the oils. The innermost pores of the monoliths may not be able to absorb oil, leading to low pore availability of the monoliths. Except for the absorption of carbon tetrachloride by microbeads, the oil-absorption ratios of both macroporous microbeads and monoliths increase with the increase of the weight fraction of internal water phase due to the increased porosity. However, the oil-absorption ratios of carbon tetrachloride by macroporous microbeads decrease with the increase of the weight fraction of $\mathrm{W}_{1}$ phase, which may also be due to the high volatility of carbon tetrachloride that made it more difficult to store in the larger pores of the microbeads. For macroporous monoliths, high porosity and large pores facilitate

Table 2 Specific surface areas and pore volumes of highly porous P(SMA-BA-St-DVB) monoliths and microbeads

\begin{tabular}{llll}
\hline & \multicolumn{2}{l}{ Specific surface area $\left(\mathrm{m}^{2} \mathrm{~g}^{-1}\right)$} & \\
\cline { 2 - 4 } Samples & $\begin{array}{l}\text { BET surface } \\
\text { area }\end{array}$ & $\begin{array}{l}\text { Langmuir surface } \\
\text { area }\end{array}$ & Pore volume $^{a}\left(10^{-3} \mathrm{~cm}^{3} \mathrm{~g}^{-1}\right)$ \\
\hline $1-\mathrm{b}-75 \%$ & 2.99 & 4.30 & 4.51 \\
$2-\mathrm{b}-80 \%$ & 3.48 & 4.87 & 5.31 \\
$3-\mathrm{b}-85 \%$ & 3.70 & 5.28 & 5.64 \\
$1-\mathrm{m}-75 \%$ & 2.79 & 3.88 & 4.94 \\
$2-\mathrm{m}-80 \%$ & 3.55 & 4.98 & 5.92 \\
$3-\mathrm{m}-85 \%$ & 3.99 & 5.69 & 6.61
\end{tabular}

${ }^{a}$ Single point adsorption total pore volume of pores less than $719.186 \AA$ diameter at $P / P_{0}=0.972$. 


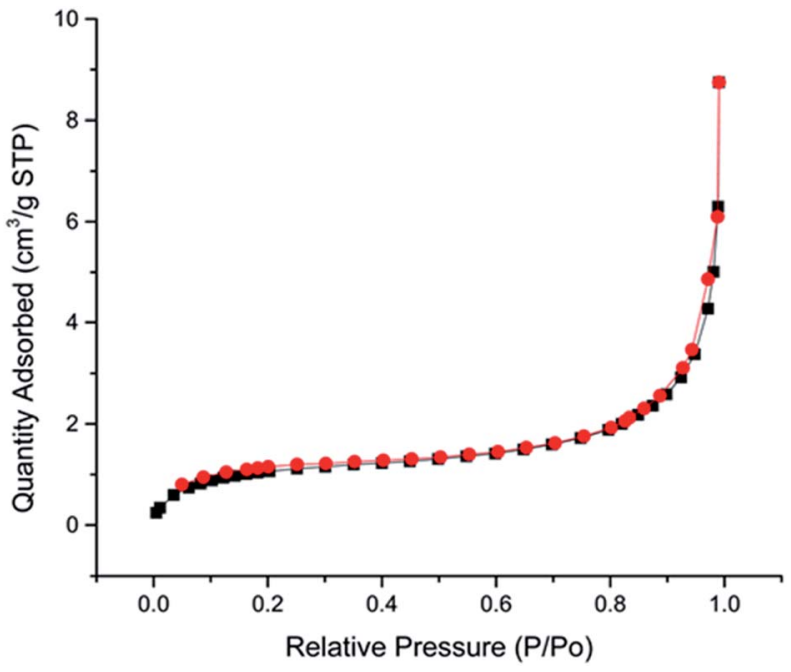

Fig. 3 Nitrogen absorption/desorption isotherm curves of sample 2b- $80 \%$.

the penetration of carbon tetrachloride into the deeper inside the monoliths, resulting in the increase of oil-absorption ratios with the increased weight fraction of internal water phase. Moreover, the oil-absorption ratios of carbon tetrachloride by both microbeads and monoliths are much higher than those of kerosene because of the higher density and the higher compatibility of carbon tetrachloride with the polymer resins. The best mass-based absorption capacity of the microbeads towards kerosene and carbon tetrachloride is 8.5 and 20.8 times of its own weight, respectively, which is similar to or even higher than those of reported polyHIPEs. ${ }^{36,37,39,41-44}$ However, these values are lower than those of reported spongy polyHIPEs, ${ }^{\mathbf{3 8 , 4 0}}$ as its porosity is much lower than those super lightweight materials as well as different polymer components.

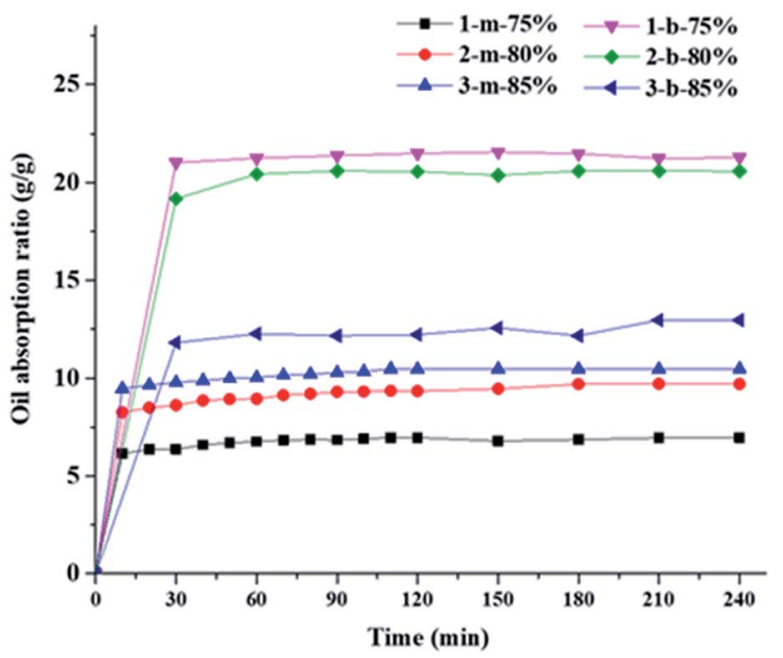

Fig. 5 Oil-absorption curves of microbeads and monolith resins to carbon tetrachloride.

\section{Thermoanalysis and oil-absorption mechanism}

The mechanism of oil absorption by absorbents is previously reported to be mainly owing to absorption, capillary action, interaction between the oils and absorbents, or a combination of these. Therefore, many parameters, such as the structure and properties of absorbents (porosity, pore size, special surface area, density, lipophilicity, etc.) and oil properties (the diffusion coefficient, viscosity, boiling point, and so on) are all governing factors of the oil-absorption capacity. Here, we mainly consider two main factors affecting oil-absorption behavior: one is the interaction between polymer skeleton and low-molecular oils, and the other is the structure of oil absorbents. The oil absorbents used in this work have highly interconnected porous structure and high porosity that enables fast mass transport and enhances oil storage capacity.

The interaction of polymer and oils could be proved by thermoanalysis. The TG-DTG curves and DSC curve of sample 2-
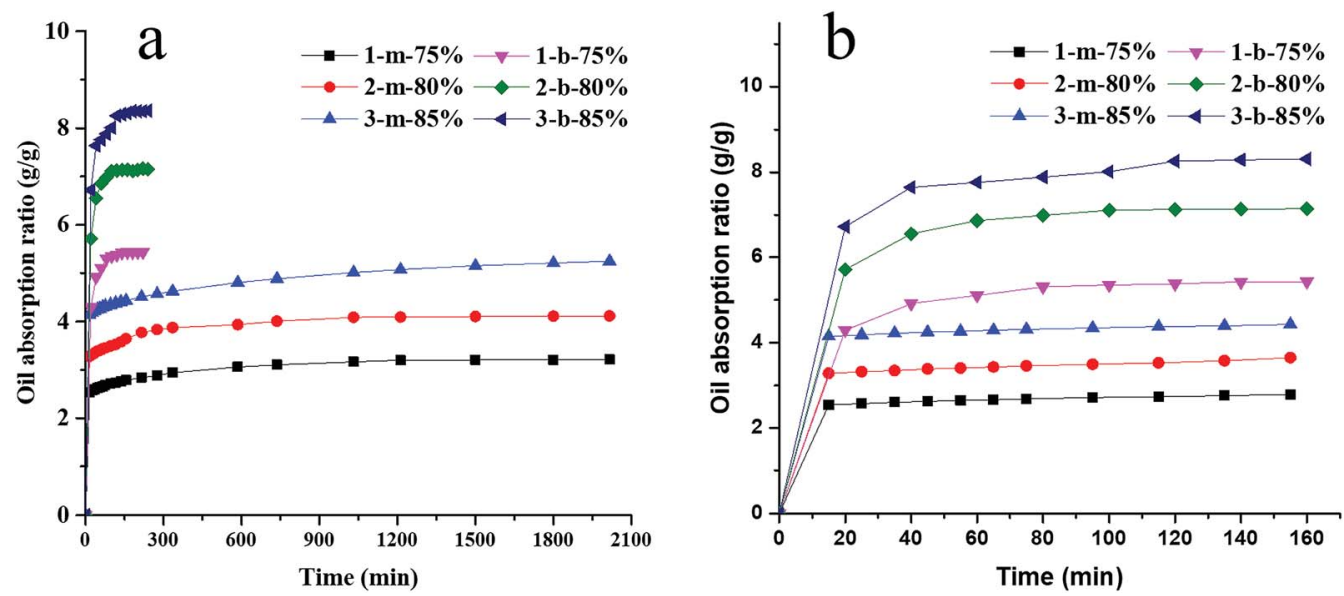

Fig. 4 Oil-absorption curves of macroporous microbeads and monoliths to kerosene: (a) complete curves of oil-absorption, (b) oil-absorption curves of the first $160 \mathrm{~min}$. 
Table 3 Oil-absorption ratios of different microbead and monolith resins to kerosene and carbon tetrachloride

Oil-absorption ratio $\left(\mathrm{g} \mathrm{g}^{-1}\right)$

\begin{tabular}{llr} 
Samples & Kerosene & \multicolumn{1}{c}{$\mathrm{CCl}_{4}$} \\
\hline 1-b-75\% & $5.23 \pm 0.06$ & $20.82 \pm 0.21$ \\
2-b-80\% & $7.29 \pm 0.08$ & $18.38 \pm 0.19$ \\
3-b-85\% & $8.52 \pm 0.10$ & $11.73 \pm 0.14$ \\
1-m-75\% & $3.21 \pm 0.04$ & $7.00 \pm 0.08$ \\
2-m-80\% & $4.08 \pm 0.06$ & $9.74 \pm 0.11$ \\
3-m-85\% & $5.34 \pm 0.06$ & $11.24 \pm 0.13$
\end{tabular}

b-80\% absorbed with kerosene as well as the TG-DTG curves of sample $2-\mathrm{b}-80 \%$ for comparison are shown in Fig. 6 . The TGA curve of sample 2-b-80\% presents three weight losses (Fig. 6(A)), which can be attributed to the pyrolysis of residual solvent and Span 80, and crosslinked P(BA-SMA-St-DVB). The second stage decomposes from $250{ }^{\circ} \mathrm{C}$ to $413{ }^{\circ} \mathrm{C}$ with about $65.5 \%$ weight loss. First, large molecular chains are randomly broken into shorter ones, and then these shorter chains randomly split to form free radicals. Moreover, the pyrolysis of residual highcrosslinked part is around $413-510{ }^{\circ} \mathrm{C}$ as a crosslinked multicomponent polymer. After absorption of kerosene, the TGA curve presents a first large weight loss of $89.1 \%$ from $20{ }^{\circ} \mathrm{C}$ to $174{ }^{\circ} \mathrm{C}$ (Fig. 6(B)), which is attributed to the desorption of kerosene.

At the same time, the weight loss of $89.1 \%$ confirms that the oil-absorption capacity of sample $2-\mathrm{b}-80 \%$ to kerosene is about $8.2 \mathrm{~g} \mathrm{~g}^{-1}$, which is consistent with the result of Table 3 within the range of experimental error. From the DSC curve, it can be easily found that there are two main endothermic peaks in the range of $20{ }^{\circ} \mathrm{C}$ to $206{ }^{\circ} \mathrm{C}$, which indicates that there are two forms of absorption mechanisms, namely physisorption (physical absorption) and swelling absorption. And TGA curve and the corresponding DTG curve show that most of the weight loss occurs at the desorption stage of swelling absorption, demonstrating that the absorption of kerosene by polyHIPE microbeads is mainly attributed to kerosene swollen in porous polymer network, namely swelling absorption mechanisms. Moreover, a slight mass loss of $7.9 \%$ is observed above $360{ }^{\circ} \mathrm{C}$, which is related to the decomposition of polymer network. The multistage degradation curve is not shown like pure polymer samples perhaps due to the small amount of polymer. It's worth noting that the thermal stability indicates that these oil absorbents can meet the demand under normal conditions up to $320{ }^{\circ} \mathrm{C}$.

\section{Oil-retention ratio}

Oil-retention ratio was characterized by the weight loss caused by oil volatilization as a function of time at a $40{ }^{\circ} \mathrm{C}$ electric blast drying oven, and the results are listed in Table 4 . It can be seen that the resins have good oil-retention properties to kerosene (more than 96\% retention after warming for $30 \mathrm{~min}$ ), while the oil-retention to carbon tetrachloride is poor (less than $50 \%$ retention after warming for 15 min except sample of 3-m-85\%), which could be attributed to the high volatility of carbon tetrachloride. These results also confirm the oil-absorption mechanism inferred above. In addition, the oil-retention ratio of microbeads to carbon tetrachloride is lower than that of the corresponding monoliths due to easy evaporation of carbon tetrachloride from large pores of microbeads, which is consistent with the above results of oil-absorption ratios of carbon tetrachloride by microbeads.

\section{Cleanup of oil slick}

In practical applications, it always involves in cleaning up the floating oil from the surface of water. In some cases like crude oil spills, the floating oil on the water surface of is fatal for some marine organism. Oil-absorption resins can float on the water surface due to its low density, which makes it easy to collect. The absorption efficiency toward spilled oil slicks was tested in a simulative condition using sample of $2-b-80 \%$ to characterize the absorption ability for floating oils in this work. When the amount of floating oil equals to $80 \%$ of total oil-absorption capacity, these microbeads can absorb the oil completely in about $10 \mathrm{~min}$. The digital photo (Fig. 7(a)) show that the yellow
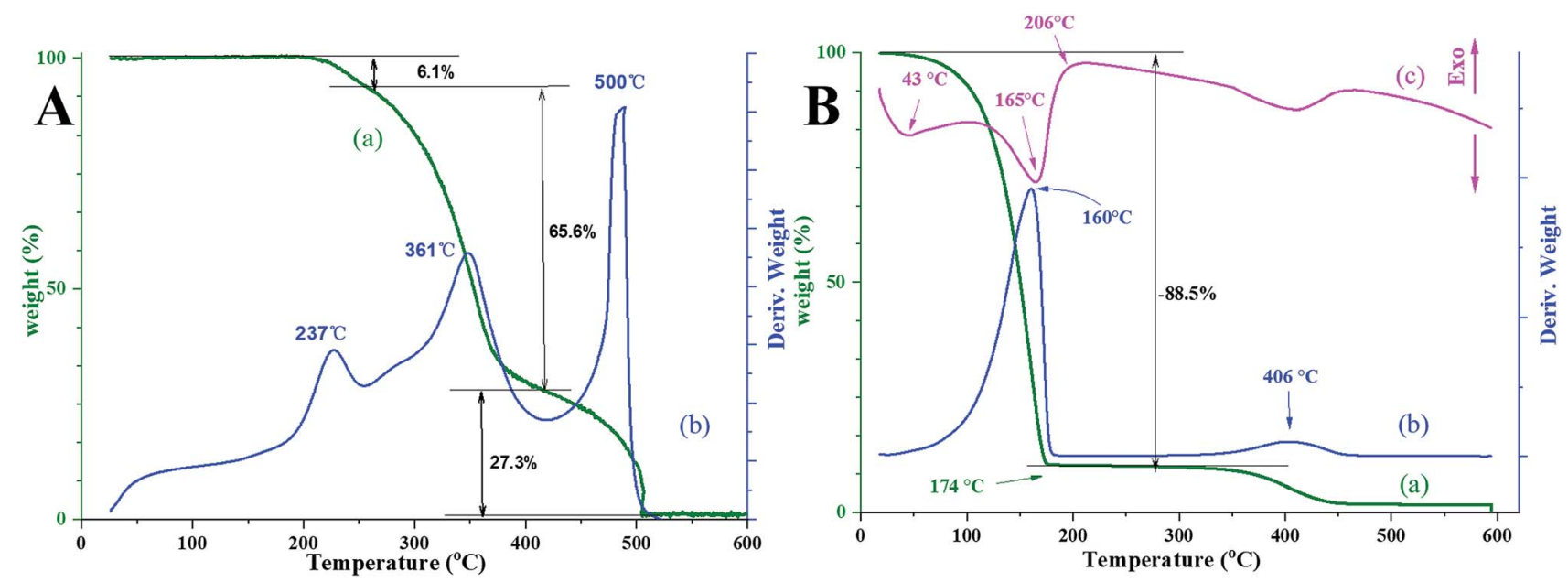

Fig. 6 Thermoanalysis of (A) 2-b-80\% sample and (B) 2-b-80\% sample absorbed with kerosene: (a) TGA curve, (b) DTG curve, (c) DSC curve. 
Table 4 Oil retention ratio of monoliths and microbeads to kerosene and $\mathrm{CCl}_{4}$

\begin{tabular}{|c|c|c|c|c|c|c|}
\hline Samples & \multicolumn{6}{|c|}{ Oil retention ratio (\%) } \\
\hline $1-\mathrm{m}-75 \%$ & $99.2 \pm 1.0$ & $98.9 \pm 0.9$ & $98.3 \pm 0.9$ & $79.1 \pm 0.7$ & $49.1 \pm 0.4$ & $9.7 \pm 0.1$ \\
\hline $2-m-80 \%$ & $99.1 \pm 0.9$ & $98.6 \pm 1.2$ & $97.9 \pm 1.1$ & $84.6 \pm 0.8$ & $48.1 \pm 0.4$ & $5.9 \pm 0.1$ \\
\hline $2-b-80 \%$ & $99.1 \pm 1.0$ & $97.2 \pm 1.0$ & $96.9 \pm 0.8$ & $47.4 \pm 0.5$ & $10.0 \pm 0.1$ & $4.5 \pm 0.1$ \\
\hline $3-b-85 \%$ & $99.9 \pm 0.9$ & $98.8 \pm 0.9$ & $97.4 \pm 1.0$ & $36.7 \pm 0.4$ & $17.7 \pm 0.2$ & $4.9 \pm 0.1$ \\
\hline
\end{tabular}
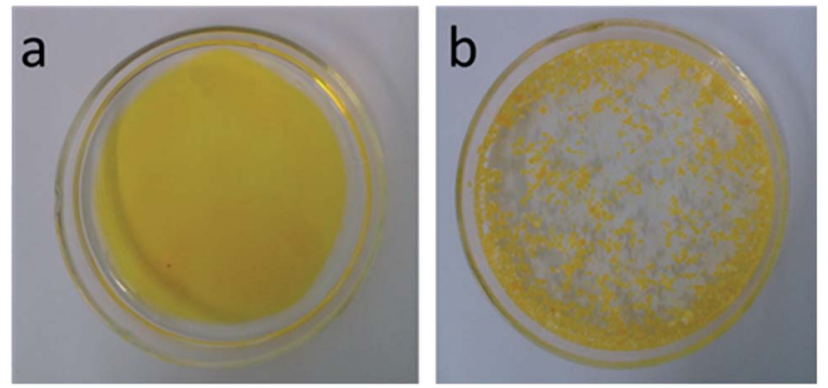

Fig. 7 The digital photos of cleanup efficiency toward simulative oil slicks (kerosene dyed with a dye of Sudan II for clarity) on the surface of water by using sample of 2-b-80\%: (a) a mixture of kerosene and water, (b) after the addition of oil absorption microbeads for $10 \mathrm{~min}$, the oil was completely absorbed by the microbeads.

oil (kerosene dyed with a dye of Sudan II for clarity) floats as a visible oil layer on the surface of water. While stirring $10 \mathrm{~min}$ with a glass rod after feeding with the microbeads, no obvious oil layer can be detected by naked eyes (Fig. 7(b)). The yellow microbeads that soaked up oil still kept floating on the surface of water, which is convenient for refloatation and after treatment.

\section{Conclusions}

In this study, we presented a method of combination of high internal phase emulsions and double emulsions as template to fabricate P(SMA-BA-St-DVB) microbeads using different weight fraction of internal water phase for serving as oil-absorption resins. The as-prepared microbeads with a typical polyHIPE structure showed better oil-absorption properties than the corresponding polyHIPE monoliths. The oil-absorption capacity of macroporous microbeads to kerosene could be as high as $8.52 \mathrm{~g} \mathrm{~g}^{-1}$, and the oil retention ratio could be more than $96.3 \%$ at $40{ }^{\circ} \mathrm{C}$ for $30 \mathrm{~min}$. Meanwhile, the oil-absorption capacity of macroporous microbeads to carbon tetrachloride could be as high as $20.82 \mathrm{~g} \mathrm{~g}^{-1}$. Thermoanalysis demonstrated that there are two absorption mechanisms, namely physisorption (physical absorption) and swelling absorption, in which swelling adsorption is dominant. Moreover, the resins showed good thermal stability as well as simple and convenient cleanup of floating oil slick, which could be promising in solution of crude oil leakage.

\section{Conflicts of interest}

There are no conflicts to declare.

\section{Acknowledgements}

We thank the National Natural Science Foundation of China (Project No. 50873096, 51373160, 21074122 and 51003122) for financial support.

\section{References}

1 J. W. Short, Energy Sources, 2003, 25, 509-517.

2 B. Dubansky, A. Whitehead, J. T. Miller, C. D. Rice and F. Galvez, Environ. Sci. Technol., 2013, 47, 5074-5082.

3 O. S. H. Santos, M. Coelho da Silva, V. R. Silva, W. N. Mussel and M. I. Yoshida, J. Hazard. Mater., 2017, 324, 406-413.

4 B. Wang, W. Liang, Z. Guo and W. Liu, Chem. Soc. Rev., 2015, 44, 336-361.

5 S. Gupta and N. H. Tai, J. Mater. Chem. A, 2016, 4, 1550-1565. 6 M. O. Adebajo, R. L. Frost, J. T. Kloprogge, O. Carmody and S. Kokot, J. Porous Mater., 2003, 10, 159-170.

7 L. Mu, S. Yang, B. Hao and P. C. Ma, Polym. Chem., 2015, 6, 5869-5875.

8 V. Broje and A. A. Keller, Environ. Sci. Technol., 2006, 40, 7914-7918.

9 J. Schaum, M. Cohen, S. Perry, R. Artz, R. Draxler, J. B. Frithsen and L. Phillips, Environ. Sci. Technol., 2010, 44, 9383-9389.

10 J. Aurell and B. K. Gullett, Environ. Sci. Technol., 2010, 44, 9431-9437.

11 R. Boopathy, S. Shields and S. Nunna, Appl. Biochem. Biotechnol., 2012, 167, 1560-1568.

12 R. M. Atlas, Mar. Pollut. Bull., 1995, 31, 178-182.

13 E. B. Kujawinski, M. C. Kido Soule, D. L. Valentine, A. K. Boysen, K. Longnecker and M. C. Redmond, Environ. Sci. Technol., 2011, 45, 1298-1306.

14 R. Wu, B. Yu, X. Liu, H. Li, W. Wang, L. Chen, Y. Bai, Z. Ming and S. T. Yang, Appl. Surf. Sci., 2016, 362, 56-62.

15 Y. K. Ongarbayev, A. S. Belgibayeva, K. K. Kudaybergenov and Z. A. Mansurov, Eurasian Chem.-Technol. J., 2015, 17, 41-45.

16 R. Wahi, L. A. Chuah, T. S. Y. Choong, Z. Ngaini and M. M. Nourouzi, Sep. Purif. Technol., 2013, 113, 51-63. 
17 Y. Chu and Q. Pan, ACS Appl. Mater. Interfaces, 2012, 4, 24202425.

18 J. Ge, H. Y. Zhao, H. W. Zhu, J. Huang, L. A. Shi and S. H. Yu, Adv. Mater., 2016, 28, 10459-10490.

19 M. S. Silverstein, Prog. Polym. Sci., 2014, 39, 199-234.

20 A. Menner, R. Powell and A. Bismarck, Macromolecules, 2009, 39, 2034-2035.

21 P. Hainey, I. M. Huxham, B. Rowatt, D. C. Sherrington and L. Tetley, Macromolecules, 1991, 24, 117-121.

22 Y. Tunc, N. Hasirci and K. Ulubayram, Soft Mater., 2012, 10, 449-461.

23 H. Xu, X. Zheng, Y. Huang, H. Wang and Q. Du, Langmuir, 2016, 32, 38-45.

24 S. Yu, H. Tan, J. Wang, X. Liu and K. Zhou, ACS Appl. Mater. Interfaces, 2015, 7, 6745-6753.

25 E. Berber, F. Cira and E. H. Mert, Polym. Compos., 2016, 37, 1531-1538.

26 J. M. Williams, A. J. Gray and M. H. Wilkerson, Langmuir, 1990, 6, 437-444.

27 D. Mao, T. Li, H. Liu, Z. Li, H. Shao and M. Li, Colloid Polym. Sci., 2013, 291, 1649-1656.

28 A. Barbetta, N. R. Cameron and S. J. Cooper, Chem. Commun., 2000, 3, 221-222.

29 Z. Li, H. Liu, L. Zeng, H. Liu and Y. Wang, J. Mater. Sci., 2016, 51, 9005-9018.

30 M. Wickenheisser and C. Janiak, Microporous Mesoporous Mater., 2015, 204, 242-250.

31 D. B. Mahadik, H. N. R. Jung, W. Han, H. H. Cho and H. H. Park, Compos. Sci. Technol., 2017, 147, 45-51.

32 S. Livshin and M. S. Silverstein, Macromolecules, 2007, 40, 6349-6354.

33 U. Azhar, C. Huyan, X. Wan, A. Xu, H. Li, B. Geng and S. Zhang, Mater. Des., 2017, 124, 194-202.

34 G. Brusotti, E. Calleri, C. Milanese, L. Catenacci, G. Marrubini, M. Sorrenti, A. Girella, G. Massolini and G. Tripodo, Polym. Chem., 2016, 7, 7436-7445.
35 S. Kovačič, K. Jeřábek, P. Krajnc and C. Slugovc, Polym. Chem., 2012, 3, 325-328.

36 P. Jing, X. Fang, J. Yan, J. Guo and Y. Fang, J. Mater. Chem. A, 2013, 1, 10135-10141.

37 X. Chen, L. Liu, K. Liu, Q. Miao and Y. Fang, J. Mater. Chem. A, 2014, 2, 10081-10089.

38 N. Zhang, S. Zhong, X. Zhou, W. Jiang, T. Wang and J. Fu, Chem. Eng. J., 2016, 298, 117-124.

39 N. Zhang, S. Zhong, T. Chen, Y. Zhou and W. Jiang, RSC Adv., 2017, 7, 22946-22953.

40 N. Zhang, Y. Zhou, Y. Zhang, W. Jiang, T. Wang and J. Fu, Chem. Eng. J., 2018, 354, 245-253.

41 Y. Wu, T. Zhang, Z. Xu and Q. Guo, J. Mater. Chem. A, 2015, 3, 1906-1909.

42 T. Zhang, Y. Wu, Z. Xu and Q. Guo, Chem. Commun., 2014, 50, 13821-13824.

43 S. Yu, H. Tan, J. Wang, X. Liu and K. Zhou, ACS Appl. Mater. Interfaces, 2015, 7, 6745-6753.

44 Z. Guo, H. Gu, Q. Chen, Z. He, W. Xu, J. Zhang, Y. Liu, L. Xiong, L. Zheng and Y. Feng, J. Colloid Interface Sci., 2019, 534, 183-194.

45 B. H. Zhang, G. C. Hardy, M. J. Rosseinsky and A. I. Cooper, Adv. Mater., 2003, 15, 78-81.

46 H. Zhang, G. C. Hardy, Y. Z. Khimyak, M. J. Rosseinsky and A. I. Cooper, Chem. Mater., 2004, 16, 4245-4256.

47 J. W. Kim, Y. G. Joe and K. D. Suh, Colloid Polym. Sci., 1999, 277, 252-256.

48 E. H. Mert and H. Yıldırım, e-Polym., 2014, 14, 65-73.

49 A. Desforges, M. Arpontet, H. Deleuze and O. M. Monval, React. Funct. Polym., 2002, 53, 183-192.

50 M. T. Gokmen, W. Van Camp, P. J. Colver, S. A. F. Bon and F. E. Du Prez, Macromolecules, 2009, 42, 9289-9294.

51 Z. A. Alothman, A. Aqel, H. A. Al Abdelmoneim, A. Yacine Badjah-Hadj-Ahmed and A. A. Al-Warthan, Chromatographia, 2011, 74, 1-8.

52 P. B. Ouchon, J. M. Aguilera and D. L. Pyle, J. Food Sci., 2003, 68, 2711-2716. 